Official Journal of the British Institute of Non-Destructive Testing
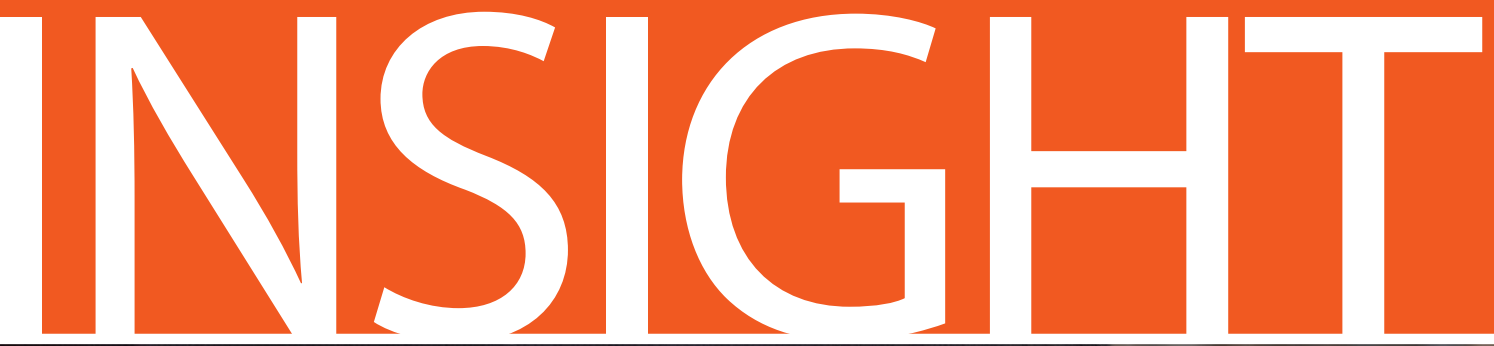

Vol 63 | № 5

May 2021

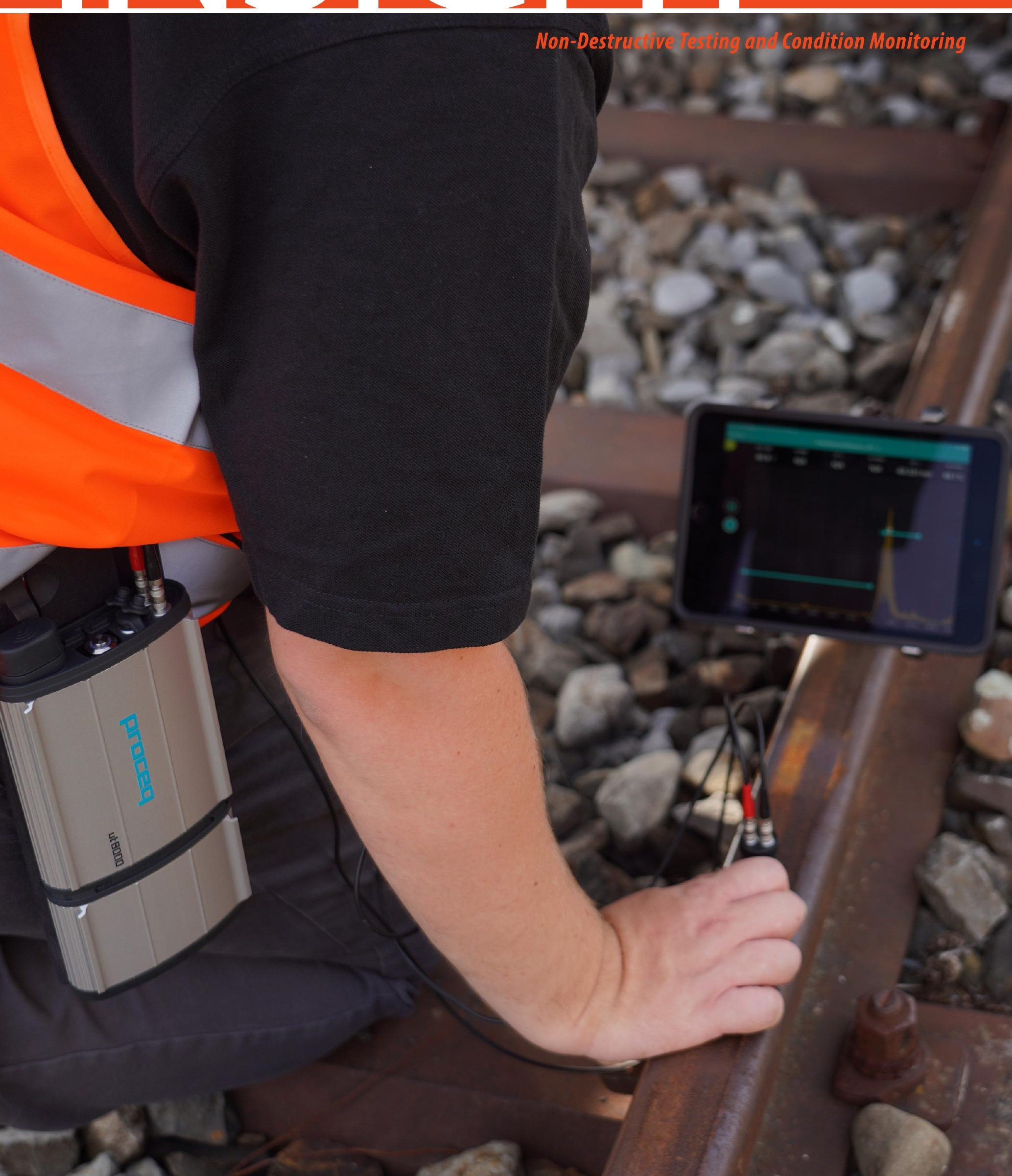




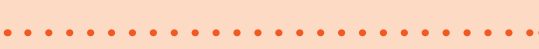

\section{This month's cover}

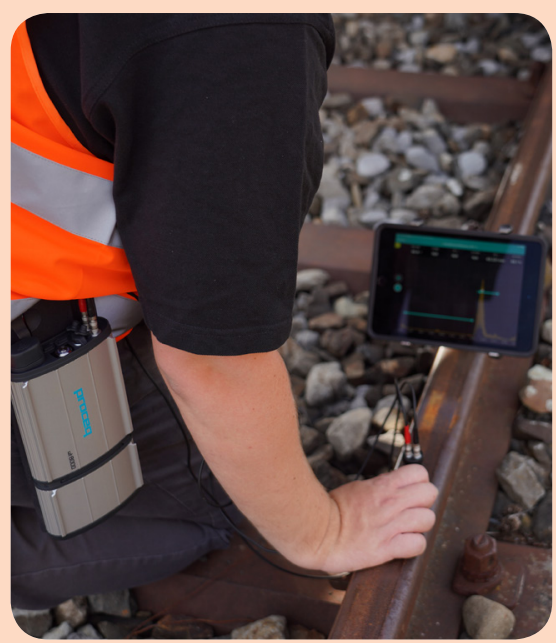

This month's front cover shows the Proceq UT8000, a portable and truly user-friendly ultrasonic testing instrument for flaw detection of metal and composite parts.

Correct, complete, precise and reusable inspection data is critical for ensuring the integrity of components and infrastructure. Proceq claims that the UT8000 represents a breakthrough in inspections with ultrasonic testing for detecting flaws such as cracks and voids in metals and composites. The rugged Swiss-made IP67-rated base unit is built for extreme levels of portability and comfort. It delivers cutting-edge bandwidth and signal-to-noise ratio, as well as allowing users to keep their existing probes and procedures.

The base unit can be connected to an iPad and its app enhances productivity with intelligent software features, such as the straightforward gesture-driven calibration procedure, the built-in indication table and the ability to rewind time for each indication. Measurements can be reviewed and findings and insights communicated and enriched with voice, photo and text annotations that deliver end-to-end traceability and peace of mind.

Proceq www.proceq.com

Published by:

The British Institute of Non-Destructive Testing Midsummer House, Riverside Way, Bedford Road Northampton NN1 5NX, UK Tel: +44 (0)1604 438300 | Fax: +44 (0)1604 438301

Email: info@bindt.org | Web: www.bindt.org

\section{NDT}

THE BRITISH INSTITUTE OF
NON-DESTRUCTIVE TESTING

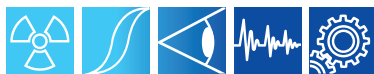

NEWSDESK: Conservation-quality 3D scanning platform for commercial art world launched; RAEng President comments on ODA and Horizon Europe funding; Teledyne e2v and Yumain announce collaboration to create AI-based imaging solutions for machine vision

\section{Special Feature: Thermography \\ LASER PULSED THERMOGRAPHY}

Depth feature extraction of surface-breaking defects using laser pulsed thermography,

by Jiaqi Liu, Zhijie Zhang, Chenyang Zhao, Ningchen Dong and Zhenyu Lin

\section{INFRARED THERMOGRAPHY}

A novel method using infrared thermography for hot fluid leakage detection on surfaces with uneven emissivities, by Xiao Zhao, Qi Zhang, Xiang Xu, Zhibin Shen and Bo Zhang

\section{Features}

\section{SHM}

Towards airborne laser Doppler vibrometry for structural health monitoring of large and curved structures,

by M Schewe, M A A Ismail and C Rembe

\section{STRESS MEASUREMENT}

Stress measurement based on magnetostrictive characteristic parameters, by Entao Yao, Fei Han, Ping Wang and Yuan Zhang......

\section{WIND TURBINE CM}

A review of the application of oil analysis in condition monitoring and life prediction of wind turbine gearboxes,

by Yu Bie, Xihao Liu, Tao Xu, Zhengfei Zhu and Zhixiong Li.

\section{Regular Features}

International Diary...

NDT Info

Product Showcase. 


\title{
Towards airborne laser Doppler vibrometry for structural health monitoring of large and curved structures
}

\author{
M Schewe, M A A Ismail and C Rembe
}

\begin{abstract}
Laser Doppler vibrometry is an important sensing technology for many structural health monitoring (SHM) methods, such as modal analysis. However, when it comes to large civil structures, for example historic structures and bridges, the applicability of laser Doppler vibrometry is significantly constrained by inaccessible remote surfaces. Some of these surfaces are fully inaccessible to a ground-mounted laser Doppler vibrometer (LDV), while others are partially inaccessible, and measurements are only possible for low incident angles. Consequently, LDV measurements are either impossible or have a weak signal strength. In this study, the concept of constructing an airborne LDV for SHM is explored, including the examination of a recently developed mechanism, the partially airborne LDV, comprising a reflective mirror attached to a drone. Preliminary proof of concept laboratory tests have been successfully conducted using two different set-ups and drone models.
\end{abstract}

Keywords: laser Doppler vibrometry, structural health monitoring, vibration analysis, drones.

\section{Introduction}

Large civil structures, for example historic structures and bridges, are typically subjected to intensive load and fluctuating environmental conditions. Such circumstances may lead to risks that influence public safety and national economies. Structural health monitoring (SHM) is aimed at mitigating these risks by assessing structural health using efficient sensing and signal processing methods ${ }^{[1,2]}$.

Recently, several SHM applications have been developed based on laser Doppler vibrometers (LDVs) ${ }^{[2-4]}$. LDVs enable non-contact vibration measurements to be performed on remote surfaces by measuring the Doppler frequency shift of a reflected laser beam (Figure 1). Hu et al ${ }^{[3]}$ utilised a long-range LDV for measuring the lateral displacement of a skyscraper. This LDV data was shown to be effective for avoiding structural damage of brittle building elements. Gioffré et al $^{[4]}$ utilised an LDV set-up for measuring the dynamic response of inaccessible tie-rods on a monumental temple.

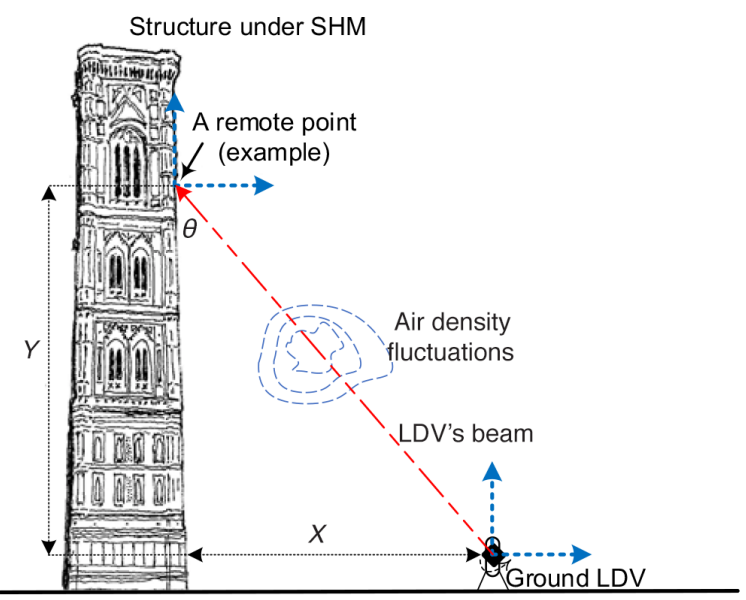

Figure 1. A common LDV arrangement for SHM of a large building. The LDV signal is subjected to significant attenuations by the low incident angle $\theta$ and the air density fluctuations
The LDV measurements were used to estimate the tensile axial forces in the tie-rods that could reflect the structural integrity and health.

However, in principle LDVs have limited applicability to large curved structures due to constraints related to fully inaccessible remote surfaces that cannot be reached by ground LDVs and partially inaccessible surfaces for which the measurements have a weak signal strength because of low incident angles ${ }^{[5]}$. As shown in Figure 1, the LDV measurements for a remote point are subjected to air density fluctuations that significantly reduce the signal strength and sensitivity. A fully airborne LDV carried by a drone has a great potential for mitigating the aforementioned limitations. However, there are many complicated problems, including aircraft noise cancellation, affecting multiple internal elements of the LDV unit ${ }^{[6]}$. Furthermore, the most compact industrial LDV on the market requires a relatively heavy drone (ie 6-10 $\mathrm{kg}$ of net payload) with stronger propeller drives and wind-gust excitations, leading to strong background noise for the LDV.

In this study, the concept of a partially airborne LDV comprising a ground-scanning LDV and a reflective mirror attached to a microdrone (for example a quadcopter) through a motorised gimbal (Figure 2) is introduced. The LDV is mounted on a motorised motion stage, allowing for the adjustment of tilt and rotation angles by a central control unit. The reflective mirrors on the drone guide the laser beam from the LDV to scan a specific area of a large structure.

\section{Submitted 02.10.20/Accepted 02.02.21}

Marvin Schewe and Christian Rembe are with TU Clausthal, Institute for Electrical Information Technology, Leibnizstraße 28, 38678 ClausthalZellerfeld, Germany.

Dr Mohamed A A Ismail* is with the German Aerospace Center (DLR), Institute of Flight Systems (FT-SSY), Lilienthalplatz 7, 38108 Braunschweig, Germany.

*Corresponding author. Tel: +49 531295 2734; Email: mohamed. ismail@dlr.de 
The reflective mirror is typically thin and light, requiring microdrones with payload capacities of only a few hundred grams. In addition, micro-drones have significantly lower vibration noise compared to heavy drones of several kilograms of net payload.

The aim of this study is to explore the measurement quality of the partially airborne LDV for internal vibration noise generated by the drone's propellers.

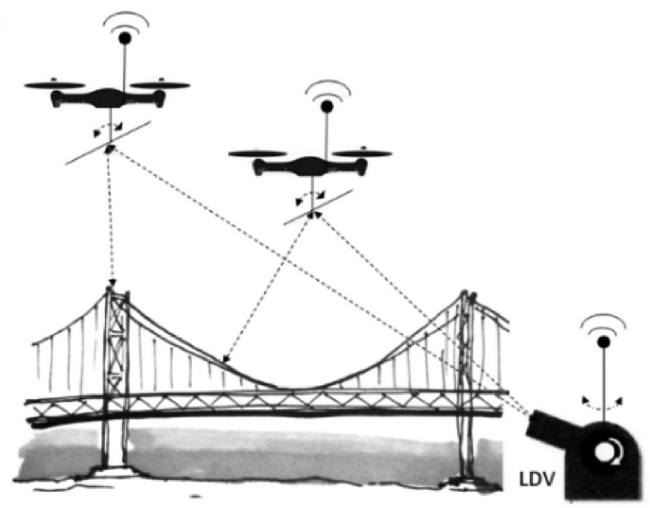

Figure 2. Partially airborne LDV comprising a ground LDV and a reflective mirror attached to a micro-drone

\section{Experimental work}

\section{Experiment 1}

The first experiment (Figures 3 and 4 ) involves an electromechanical shaker as a vibration target. An LDV, model PSV-400, is used to measure the target vibration through two transmission paths: directly (Path 1) and through a mirror attached to a drone (Path 2). The drone is a quadcopter model AR Parrot 2 and it hovers vertically above the shaker. A soft link is added between the drone's boom
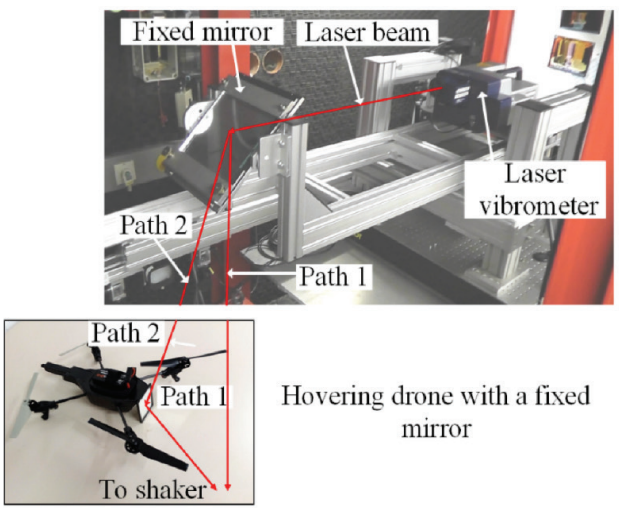

Hovering drone with a fixed mirror

Figure 3. Set-up used in Experiment 1

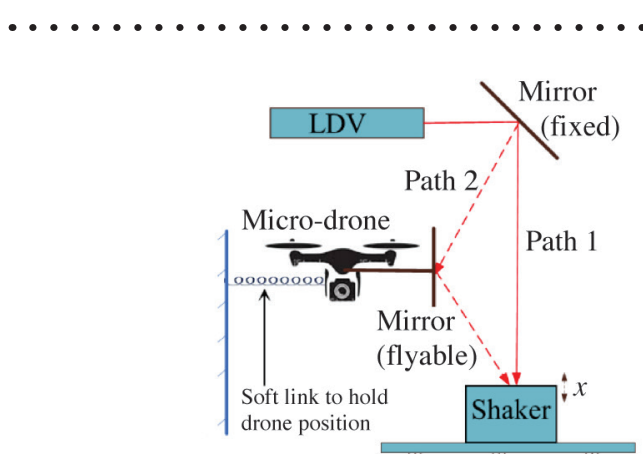

Figure 4. Schematic drawing of Experiment 1 and a stationary point to constrain the drone's position inside the lab for safety-related reasons.

Three sinusoidal low frequencies were applied to the shaker at $2 \mathrm{~Hz}, 5 \mathrm{~Hz}$ and $9 \mathrm{~Hz}$ to represent common resonance frequencies for large structures ${ }^{[1,2]}$. Fast Fourier transforms (FFTs) of the vibration measurements through Paths 1 and 2 of the LDV are shown in Figure 5. Measurements of Path 2 compensated for the tilted incident angle compared to the vertical incident angle seen in Path 1. Small measurement deviations, of $2-5 \%$ of the full scale, are observed between Paths 1 and 2 due to the drone's limited shaking. Here, the drone noise was concentrated around the blade-pass frequency of $60 \mathrm{~Hz}$, which is far from the target frequency range.

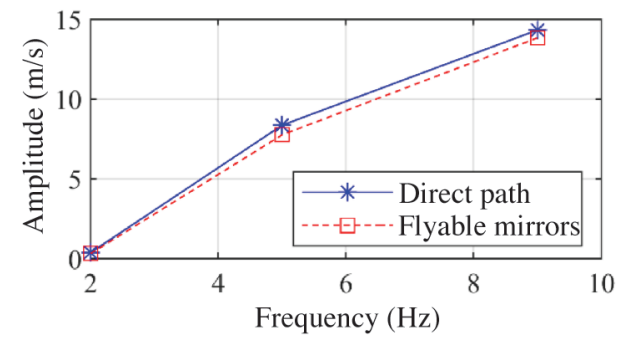

Figure 5. The vibration measurement of Experiment 1 demonstrates that the flyable mirror concept may allow accurate measurements to be performed

\section{Experiment 2}

In real-world applications, it is highly probable that the noise contribution of both the drone and various environmental influences will overlap with the target frequency range. Therefore, there is a need to reduce the noise level. One possibility for decreasing the noise level involves utilising a differential LDV (DLDV) ${ }^{[7]}$. To highlight the capability of noise reduction with a DLDV, a reflector and a mirror are attached to a larger drone (the DJI Mavic 2 Pro) with the rotors set to idle mode ( $1900 \mathrm{r} / \mathrm{min})$. Figure 6 shows the experimental set-up. The reference beam of the DLDV is targeted on the reflector, whereas the measurement beam is focused on the structure via the mirror on the drone. In this case, a speaker with a sinusoidal wave of $222 \mathrm{~Hz}$ represents the structure.

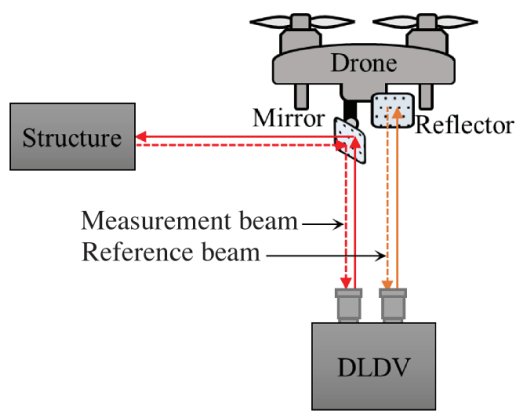

Figure 6. Schematic drawing of Experiment 2

With this set-up, the reference beam transmits the drone noise, while the measurement beam transmits both the drone noise and the vibration from the structure. Consequently, the drone noise can be compensated through the interference of both laser beams. Hence, the noise is lower in comparison to a normal LDV measurement (Figure 7). In Figure 7, both LDV and DLDV measurements have a resolution bandwidth of $156 \mathrm{MHz}$. The signals are filtered with a fifth-order median filter and the amplitude of the speaker vibration is approximately the same. 


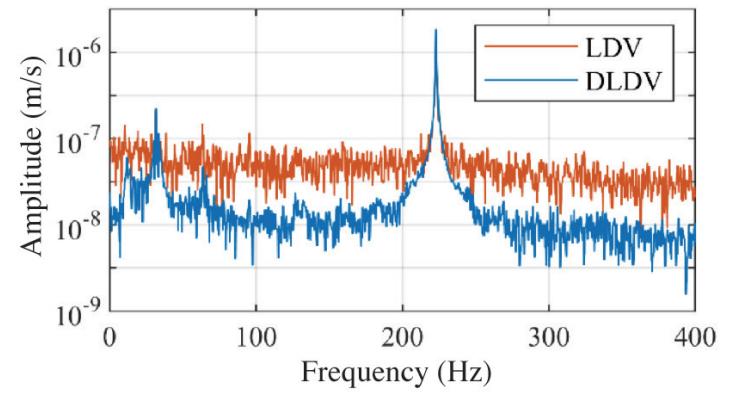

Figure 7. Vibration measurement with an LDV and a DLDV set-up to compare noise levels

\section{Conclusion}

The partially airborne LDV, comprising a ground LDV and a reflective mirror attached to a drone, has potential for evaluating large and curved structures. A crucial challenge is the drone noise, which can be isolated by band-pass filters for simple flight conditions, for example hovering at a fixed indoor position. Dynamic flight cases, including wind gusts, require an adaptive noise cancellation mechanism for continuous identification of the drone noise. In this first study, a differential LDV has been successfully utilised to reduce drone vibration noise in the vibration measurement of a target structure. Future work will examine the influence of incident angles on the drone's mirror and the broadening of the frequency range of structural vibration measurements and drone noise suppression in outdoor conditions.

\section{References}

1. D Inaudi, 'Overview of 40 bridge structural health monitoring projects', Proceedings of the 26th International Bridge Conference (IBC 2009), Pittsburgh, Pennsylvania, USA, pp 343-350, 14-17 June 2009.

2. T Miyashita and M Nagai, 'Vibration-based structural health monitoring for bridges using laser Doppler vibrometers and MEMS-based technologies', International Journal of Steel Structures, Vol 8, No 4, pp 325-331, 2008.

3. W H Hu, Z M Xu, M Y Liu, D H Tang, W Lu, Z H Li, J Teng, X H Han, S Said and R Rohrmann, 'Estimation of the lateral dynamic displacement of high-rise buildings under wind load based on fusion of a remote sensing vibrometer and an inclinometer', Remote Sensing, Vol 12, No 7, p 1120, 2020.

4. M Gioffré, N Cavalagli, C Pepi and M Trequattrini, 'Laser Doppler and radar interferometer for contactless measurements on unaccessible tie-rods on monumental buildings: Santa Maria della Consolazione Temple in Todi', Journal of Physics: Conference Series, Vol 778, No 1, p 012008, 2017.

5. M A A Ismail, 'DE102018113244: Verfahren und Vorrichtung zum Messen von Schwingungen eines Objekts unter Verwendung einer Drohne', German Patent, 2018.

6. B J Halkon and S J Rothberg, 'Towards laser Doppler vibrometry from unmanned aerial vehicles', Journal of Physics: Conference Series, Vol 1149, No 1, p 012022, 2018.

7. M Schewe, D Kohlmann, $\mathrm{H}$ Wulfmeier, $\mathrm{H}$ Fritze and C Rembe, 'Methoden zur Minimierung des Rauscheinflusses durch Hitzeflimmern bei einem heterodynen Laser-DopplerVibrometer', tm - Technisches Messen, Vol 87, No s1, pp s44s49, 2020.

\section{Workshop on multicopters for inspection}

\section{Wednesday 16 June 2021}

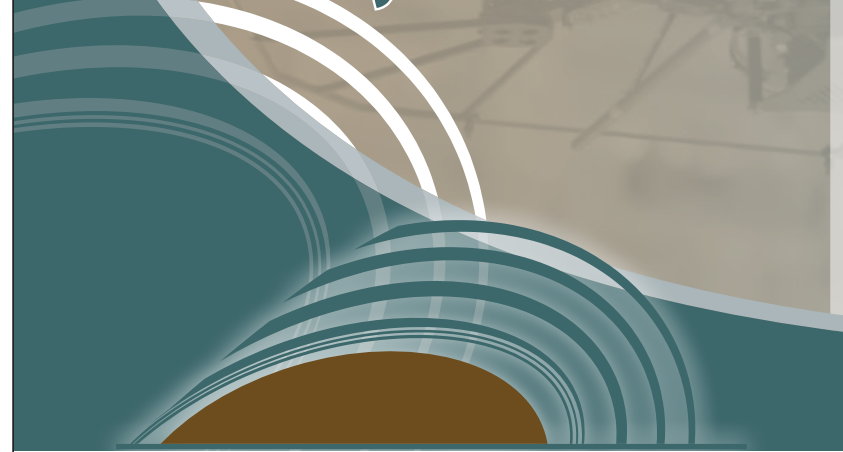
Multicopters Workshop

\section{Member of BINDT} $£ 25.00+$ VAT per session*

Non-member of BINDT $£ \mathbf{3 0 . 0 0 + V A T}$ per session* savings have been made through the access or hostile to human inspectors. Great cost inspection methods that often require scaffolding to be erected.

Drones can carry high-resolution cameras, thermal imaging equipment or light detection and ranging (LIDAR) equipment. Recent developments include the deployment of ultrasonic sensors to take thickness measurements of remote components.

The aim of the workshop is to inform delegates of the legal, safety and training requirements, supported by case studies of a variety of applications, including oil \& gas, wind turbines, rail, power generation and distribution.

*The webinar may be one or two sessions depending on accepted presentations.

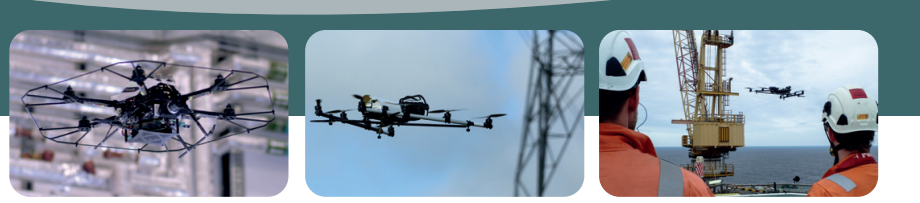

Contact: Conferences and Events Department The British Institute of Non-Destructive Testing, Midsummer House, Riverside Way, Bedford Road, Northampton NN1 5NX, UK

Tel: +44 (0)1604 438300; Fax: +44 (0)1604 43830 Email: conf@bindt.org; Web: www.bindt.org 